\title{
Análisis comparativo en el mapeo de terreno usando Vehículos Aéreos No Tripulados con relación al método convencional en la localidad de Piruruyoc - Ancash
}

Comparative analysis of the terrain mapping using Unmanned Aerial Vehicles in relation to Conventional Method in the locality of Piruruyoc - Ancash

Yoshiro Crhistian Zuñiga Flores ${ }^{1}$

\section{RESUMEN}

La presente investigación ha tenido como objetivo analizar la «exactitud, el tiempo y el costo» del levantamiento de una nube de puntos topográficos elaborada a partir del uso de Vehículos Aéreos No Tripulados (VANT) comparada con el método convencional con el uso de una estación total. La Metodología de comparación se basó en las directrices de la Norma de Exactitud Posicional en Datos Geoespaciales de la «Sociedad Americana de Fotogrametría y Teledetección». La captura de los datos fotogramétricos se realizó con un drone de Ala Rotatoria Phantom 3 Profesional a $120 \mathrm{~m}$ de altura sobre el terreno a un traslape (Longitudinal/Transversal) 80/75. El proceso de datos se realizó con el software Agisoft PhotoScan 2015 que favoreció obtener 3.32 millones de puntos $3 \mathrm{D}$ y un GSD de $5.41 \mathrm{~cm} /$ pix, resultados comparados con datos de referencia del método convencional que revelaron un Error Cuadrático Medio de $13.16 \mathrm{~cm}$ en planimetría y $13.95 \mathrm{~cm}$ en altimetría, error que según la clasificación para la obtención de datos geoespaciales, ubica los datos dentro del rango de «Mapeo Estándar/Uso para Información GIS». Se concluye que la tecnología VANT o Drone aplicada en el mapeo de terreno es de uso confiable en proyectos de obras civiles, información de considerable importancia para propósitos de evaluación de terreno y de mayor incidencia en etapas tempranas en el desarrollo de proyectos de ingeniería.

Palabras clave: vehículo aéreo no tripulado; drone; mapeo de terreno, fotogrametría; ingeniería civil.

1 Universidad Nacional Santiago Antúnez de Mayolo. Huaraz, Perú. 


\section{ABSTRACT}

This research has aimed to analyze the «accuracy, time and cost» in the topography's work; the creation of point's cloud through the use of Unmanned Aerial Vehicles (UAVs) compared to the conventional method with a total station. The comparison methodology was based on the guidelines of the Positional Accuracy Standards for Digital Geospatial Data of the «American Society for Photogrammetry and Remote Sensing» (ASPRS, 2014). The UAV used was a Phantom 3 Professional rotary wing. The images were acquired from a height of 120 meters with an overlap of 80/75 (longitudinal/transverse). The data was processed with the Software Agisoft PhotoScan 2015 obtained 3.32 millions of 3D points and $5.41 \mathrm{~cm} /$ pix GSD. The results compared to the conventional method revealed a $13.16 \mathrm{~cm}$ error in planimetry and $13.95 \mathrm{~cm}$ in altimetry, within the range of «Standard Mapping / GIS Use for information» by the classification of digital geospatial data of the ASPRS, 2014. The analysis concludes that the UAVs technology applied for mapping land has a reliable use in engineering's projects, information of considerable importance for the purposes of evaluation of land, and with highest incidence in early stages of the development of engineering's projects (Blake, 1989: 7.8).

Keywords: unmanned aerial vehicle; drone; terrain mapping, photogrammetry; civil Engineering.

\section{ICHIKLLACHAW}

Kay musyapakuy ashipakuychawqa kapachninta, patsayninta, chanintam imanawmi patsachaw pukutay shaariynintam alliq qawakashqa, kayqa imanawmi rara wayrapa llapan mana runayuq puriynintam riman, kayqa VANT nishqanmi. Kay musyapakuyqa ñawpa riqishqana miituwanmi qaqantsaqashqa tsullantsakashqa. Kaypaqqa paqway istasiyun nishqantam wanakashqa. Kay tsullankashqa mituduluhiiyaqa «Sociedad Americana de Fotogrametría y Teledetección (ASPRS, 2014) nishqanwanmi pallarikashqa, kaykunawanran patsaypa llapan aywayninpis tinkukashqa. Kay hutugramitriku nishqan tsarishqanqa Dronewan riprapa kuyuy Phantom nishqanwanmi rurakashqa, kayqa 3piq 120m-kama patsapiq rarakama awqaynin aywayninmi, kay tsalapi nishqanqa (karu kinrayninwan llapan witsayninqa) 80/75kamam chan. Kay llapan rurayninqa Software Agisoft PhotoScan 2015wanmi rurakashqa, kaywanmi 3.32. hunukunata awqaqashqa 3Dpa chikunkunata. hinaman huk GSD $5.41 \mathrm{~cm} /$ pixkama awqaqanqantam. Kay llapan hurqakashqaqa nawpa riqishqa miituduwanmi qaqantsashqa, tsayraykurmi mana alli Kwadratiku Pullan $13.16 \mathrm{~cm}$ planimitriya nishqan yarqushqa, hinaman 13.95 cm altimitriya nishqanmi yarqushqa. Tsay llapanqa 2014chaw ASPRSpa mana alli yarquyninmi, tsayraykurmi kay llapan «Mapeo Estándar nishqan hinaman Willakuypaq rurashqa GIS nishqan rurinchaw yarkushqa. Tsay llapan nishqan VANTpis Drone nishqanpis patsachaw mapiyu «Siwil Ruray Pruyiktu» hutishqa ruraynichaw allim 
yarqushqa, tsayraykurmi llapan ashipakuy willakuyninqa allaapa iñinam, inhiñiriyapa pruyiktu rurayninchawnaaqa kay punta kaq yuriyninaaqa allaapa allim.

Pushaq shimikuna: mana runawam puriq wayra; drone nishqan; patsapa mapiyun; hutugramitriya; inhiñiriya siwil nishqan.

\section{INTRODUCCIÓN}

El uso de la fotogrametría ha tenido un gran impulso en la actualidad debido al avance vertiginoso del progreso tecnológico, y más aún la miniaturización de la tecnología VANT (Vehículos Aéreos no Tripulados) o Drones, los cuales, al ser equipados con sistemas de Posicionamiento Global, Unidades de Medición Inercial (IMUs), sensores de proximidad y sensores fotográficos de amplias resoluciones, hacen posible una plataforma estable y eficiente para la toma de datos fotográficos aéreos. Datos que, al ser capturados con una metodología específica y procesados por potentes algoritmos informáticos de restitución fotogramétrica, hacen posible la generación de miles de millones de puntos georreferenciados en el espacio, mejorando la productividad en el análisis de la superficie terrestre.

En Huaraz, la fotogrametría, de muy poco uso en nuestro entorno, es una disciplina potente capaz de proporcionar una nube de puntos geo-referenciados en el espacio que poseen propiedades de color y textura que abren nuevas fronteras para el análisis del espacio territorial a través de sistemas SIG, no solo en la toma de datos topográficos enfocada al diseño en infraestructura, sino para el monitoreo constante de determinado espacio con enfoque a la supervisión de obras, apoyo en la solución de controversias, planeamiento. En cuanto a la prevención de desastres naturales, soporte en la mejora del tiempo de respuesta ante situaciones de desastre, apoyo para el diseño de ingeniería en la identificación de características físicas y geológicas del terreno, análisis de flujos de agua, escorrentía, diseño hídrico, identificación de recursos naturales, entre otros usos funcionales.

El estudio tiene relevancia debido a que la metodología fotogramétrica con drones todavía no ha sido probada en nuestro medio académico, ni difundida, pues resulta ser de vital importancia para el desarrollo de nuevos enfoques en la ingeniería; por todo ello ha surgido la necesidad académica de aplicar la técnica fotogramétrica en nuestro entorno con los nuevos avances tecnológicos y comparar los resultados con el método convencional de uso común en nuestra localidad y demostrar que se puede producir resultados fiables de aplicación potencial en proyectos de ingeniería.

\section{MATERIALES Y MÉTODOS}

El estudio se inicia con la obtención de los parámetros básicos para la toma de datos aéreos apropiados con las características y restricciones propias del vehículo aéreo no 
tripulado, tales como el GSD, la separación entre ejes del vuelo, tiempo de captura entre fotografías y la velocidad de viento segura. Con los datos calculados se elaboró el plan del vuelo adecuado, cubriendo un área de toma de datos de 15.50 hectáreas. Se cargó las coordenadas en el programa de vuelo automatizado (Aplicación Litchi 2.6.1.). En la zona de estudio se eligió los puntos de interés más representativos y «visibles» desde la altura para ser utilizados como puntos de verificación entre las dos metodologías, se identificó 04 Puntos de Control Terrestre (GCP) para realizar el ajuste de restitución fotogramétrica, y 27 Puntos de Verificación (CP) para fines de comparación respecto a ambas metodologías; se tomaron datos de campo con el vehículo aéreo no tripulado y con la estación total. Luego se procedió a procesar la información en el software de restitución fotogramétrica Agisoft PhotoScan 2015 y Civil 3D 2016, respectivamente.

El análisis de exactitud en planimetría y altimetría siguió los lineamientos de la Norma de Exactitud Posicional en Datos Geoespaciales de la «Sociedad Americana de Fotogrametría y Teledetección» (ASPRS, 2014), el cual establece un mínimo de 20 puntos de verificación del conjunto de geodatos (dataset), con un equipo de mayor exactitud, en nuestro caso la estación total $(+/-2 \mathrm{~mm})$; comparada con los datos obtenidos por el drone para idénticos puntos, finalmente, se determinó el error cuadrático medio (RMSEx) aplicando la técnica de análisis numérico «mínimos cuadrados» a través de la siguiente expresión:

$$
\begin{gathered}
\text { RMSE }=\sqrt{\frac{1}{n} \sum_{i=1}^{n}[P i(\text { Fotogrametría })-P i(\text { Estación Total })]^{2}} \\
\text { Donde: Pi }=\text { Punto en la coordenada especificada }(\mathrm{X}, \mathrm{Y} \text { o Z })
\end{gathered}
$$

En cuanto al análisis del tiempo y costo, el mencionado análisis se efectuó con la información recabada para cada metodología según proceso de campo y gabinete.

La población la constituye el área de 15.5 ha, tomada para el presente estudio. La unidad de análisis y muestra está constituida por la misma población.

En cuanto a los equipos para la investigación, se utilizó un Vehículo Aéreo no Tripulado de la marca DIY Modelo Phantom 3 Profesional de ala rotatoria, una Estación Total Sokkia 530RK con exactitud y con prisma +/- 2 mm y un anemómetro digital.

\section{RESULTADOS}

La localidad de Piruruyoc, se encuentra en el distrito de Independencia, Provincia de Huaraz, departamento de Ancash, Perú. Ubicada en la cordillera Negra a 1 hora 50 minutos a través de la carretera Huaraz-Casma, según se observa en la vista de la figura 1. 


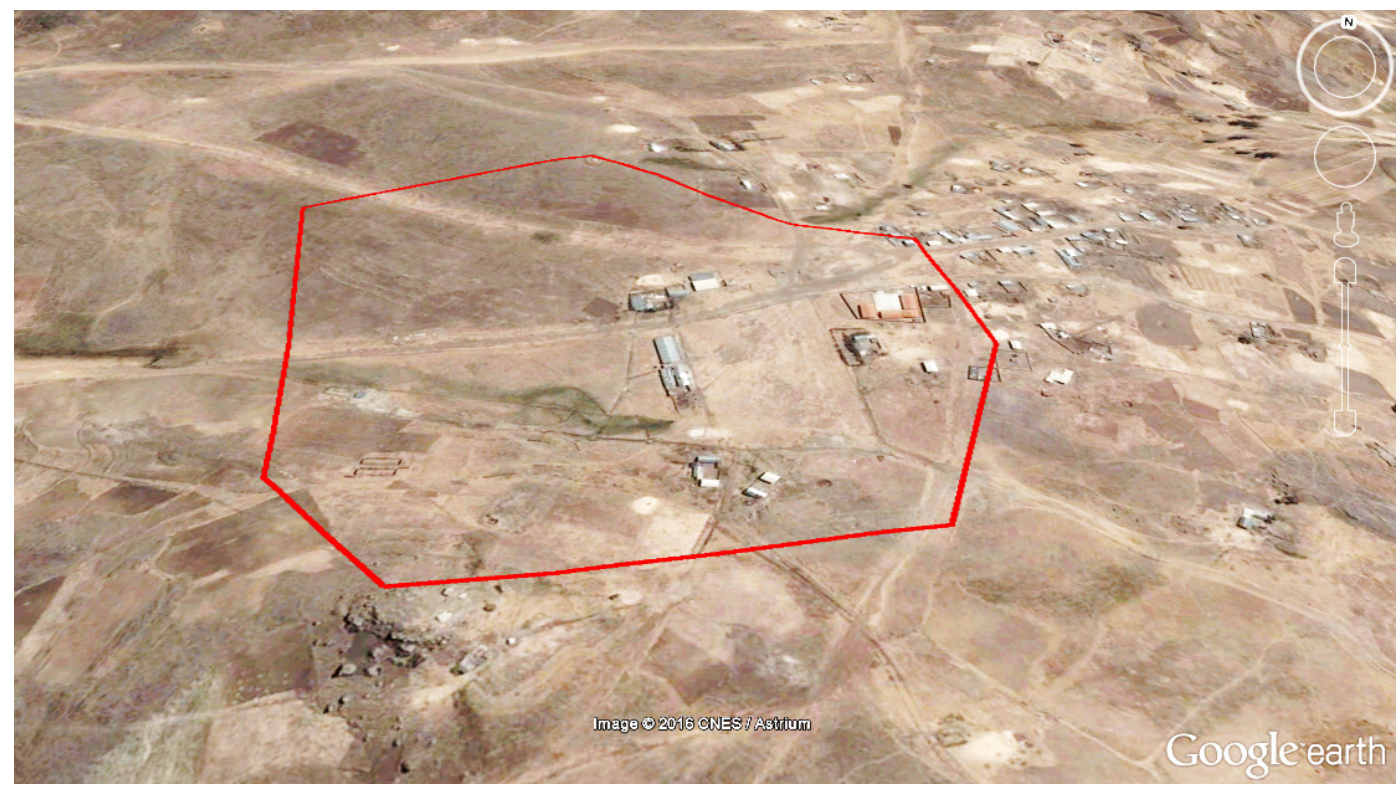

Figura 1. Vista de la zona de Estudio en Piruruyoc-Ancash

La toma de datos se realizó con el Drone Phantom 3, previo análisis de la velocidad de viento y condiciones seguras del equipo, que se puede observar en la figura 2.

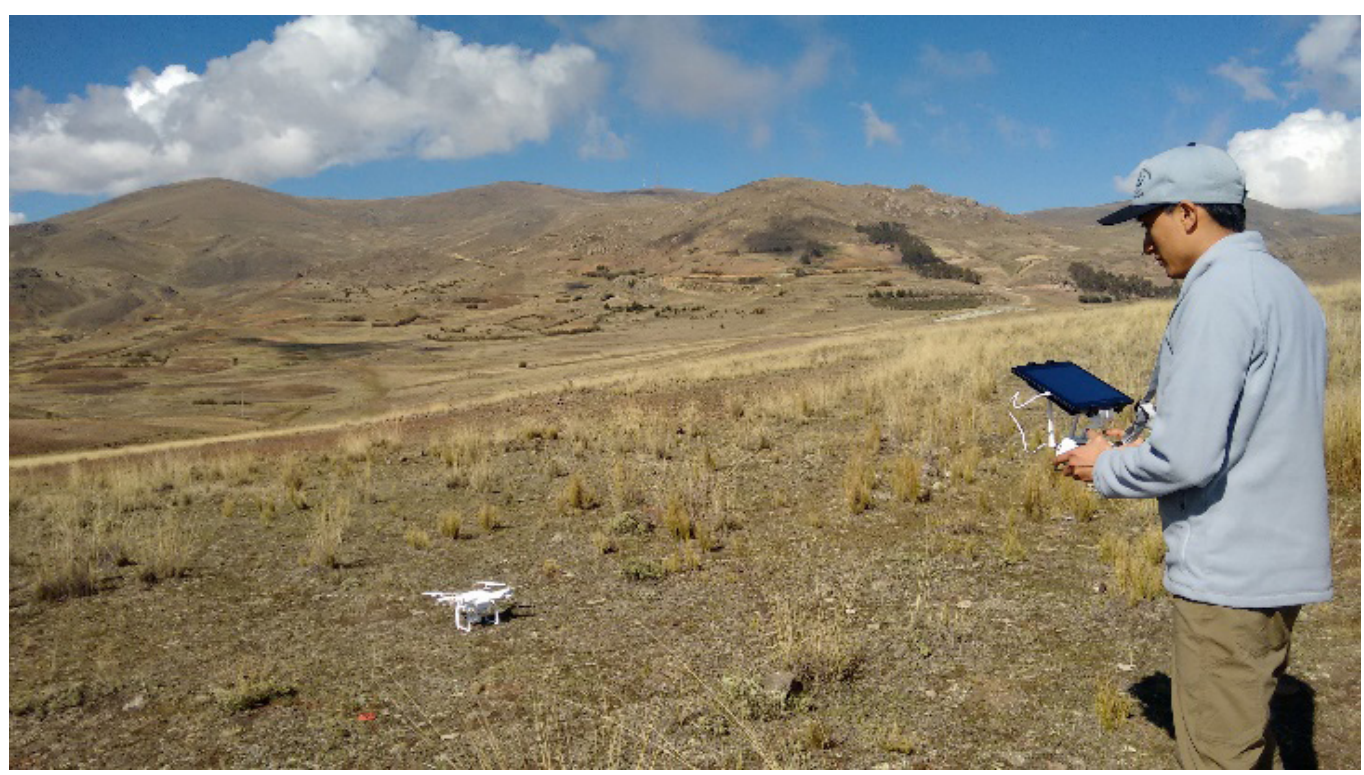

Figura 2. Drone Phantom 3 Profesional listo para toma de datos fotogramétricos

El procesamiento del modelo digital del terreno y las ortofotos se observan en la figura 3; además, en la derecha se visualiza los 27 puntos de verificación dentro del área de estudio, utilizados para la verificación del error cuadrático medio (RMSE). 

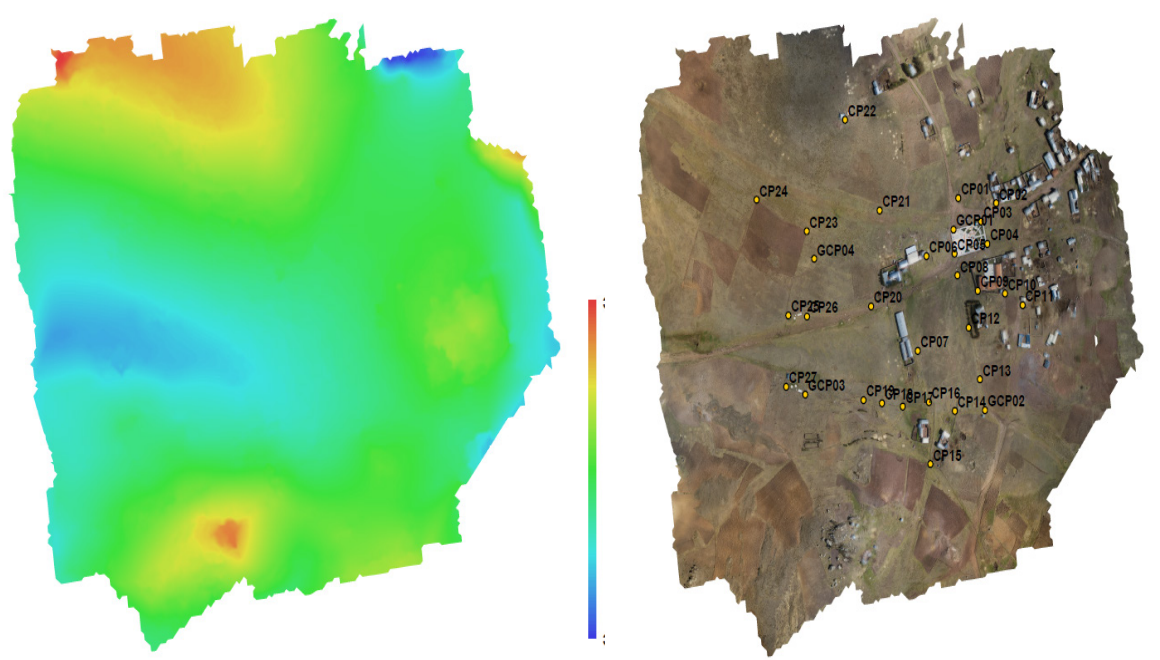

Figura 3. Modelo Digital de Terreno (derecha) y Ortofoto (izquierda)

Los resultados del procesamiento por el método convencional utilizando la estación total se observan en la figura 4. Los 27 puntos de verificación en cruz y los 4 puntos de control terrestre para ortorrectificación en triángulo dentro del área de estudio en línea punteada.

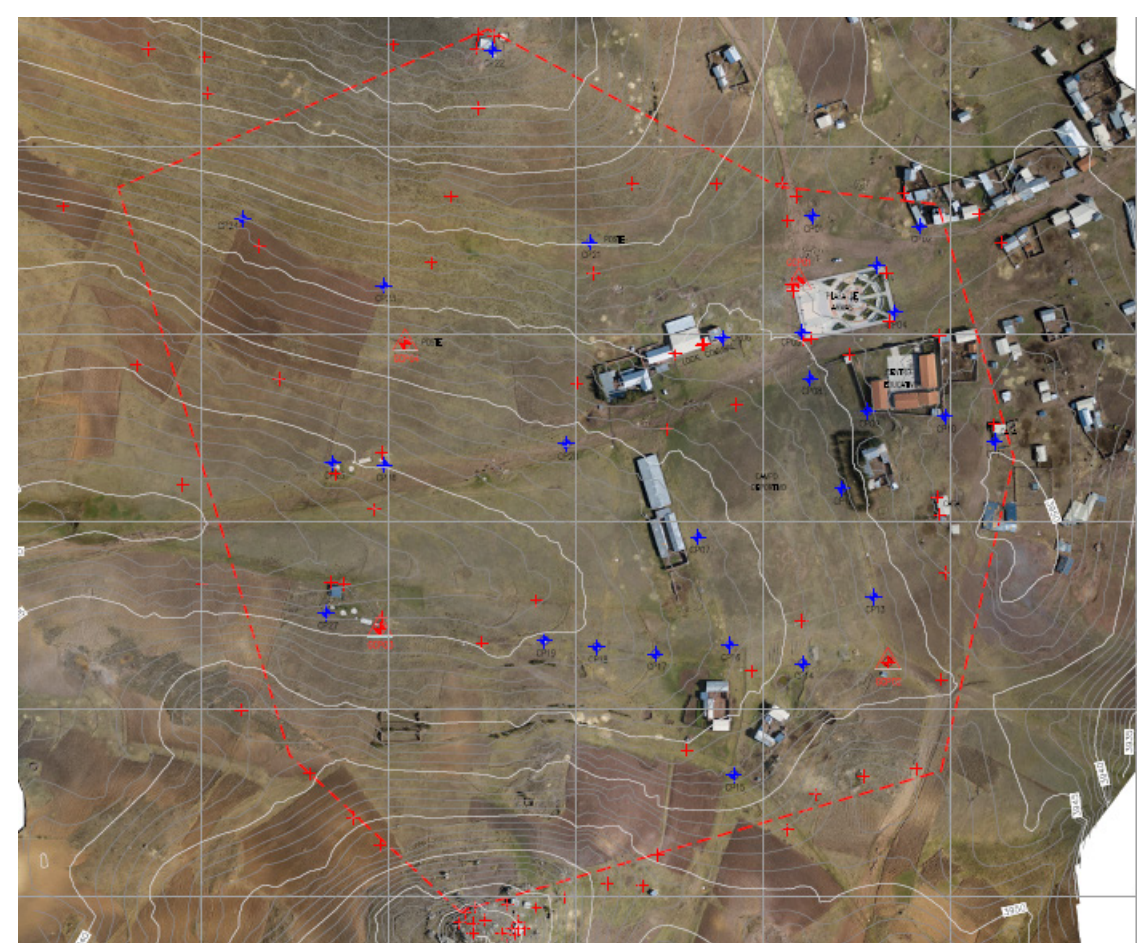

Figura 4. Los 27 Puntos de Verificación obtenidos con estación total dentro del área de estudio (línea punteada) 
Los resultados del análisis de exactitud comparativa entre la data obtenida por el drone y la estación total de las coordenadas (X, Y, Z) de los 27 puntos de verificación se observan en la tabla 1.

Tabla 1. Resultados en cuanto a exactitud en el mapeo de terreno

\begin{tabular}{lc}
\hline \multicolumn{1}{c}{ Parámetros } & Resultados \\
\hline GSD (resolución) & $5.41 \mathrm{~cm} /$ pix \\
RMSE $_{\mathrm{r}}$ Error cuadrático medio en planimetría $(\mathrm{X}, \mathrm{Y})$ & $13.16 \mathrm{~cm}$ \\
RMSE $_{\mathrm{Z}}$ Error cuadrático medio en altimetría $(\mathrm{Z})$ & $13.95 \mathrm{~cm}$ \\
\hline
\end{tabular}

En cuanto al resultado comparado con el método convencional en tiempo y costo, para la misma extensión de terreno (15.5 ha), se puede observar en las figuras 5 y 6 .

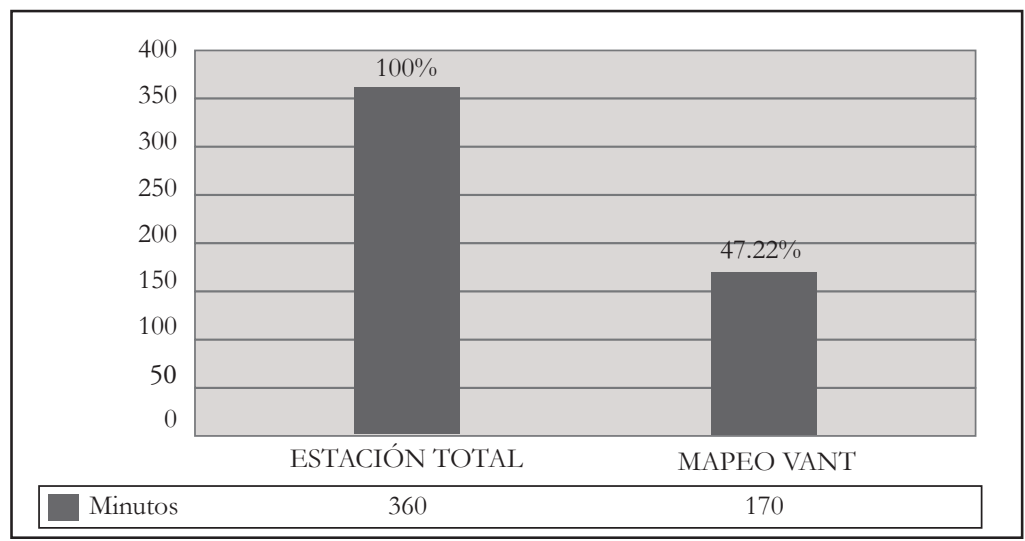

Figura 5. Datos comparados en cuanto al tiempo

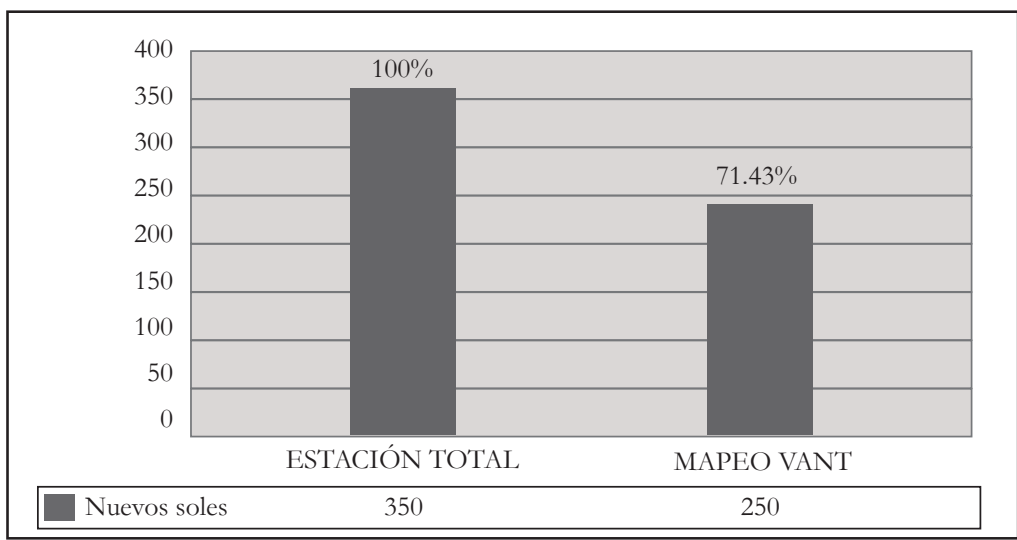

Figura 6. Datos comparados en cuanto al costo 


\section{DISCUSIÓN}

En cuanto a la exactitud en planimetría, las variaciones de exactitud encontradas respecto a Siebert y Teizer (2013), son consistentes con los valores encontrados, debido a que ellos utilizaron una altura de vuelo de $50 \mathrm{~m}$, y una cámara de mayor resolución. Según el Estándar ASPRS, en Planimetría el error RMSE r $_{\mathrm{r}}=13.16 \mathrm{~cm}$, tabla 07, se valida su uso para un «Mapeo Estándar y Trabajo GIS», especificando «Esta exactitud es apropiado para un nivel estándar de alta exactitud para aplicaciones de mapas geoespaciales de alta calidad. Es equivalente a precisiones ASPRS 1990 Clase1, según la interpretación de los usuarios como estándar de la industria y presentado en la Tabla B.4. Este nivel de exactitud es típico de una gran mayoría de los proyectos existentes.» (ASPRS 2014, A13). La exactitud en planimetría es aceptable en el mapeo de terreno utilizando vehículos aéreos no tripulados en relación con el método convencional según las ASPRS. 2014. Además, la escala encontrada es consistente con las escalas acostumbradas por la tecnología para vuelos aéreos con drones según Garcia Julián (2015).

En cuanto a la exactitud en altimetría, las variaciones respecto a Siebert y Teizer (2013), son consistentes con los valores encontrados debido a que ellos utilizaron una altura de vuelo de $50 \mathrm{~m}$, y una cámara de mayor resolución, además de que el terreno en el presente estudio presenta variaciones con vegetación de poca altura pero densa. Según el Estándar ASPRS en Altimetría el error RMSE $\mathrm{z}=13.95 \mathrm{~cm}$, en la tabla 10, las ASPRS 1990 clase 1, son equivalentes a $45 \mathrm{~cm}$ de exactitud de contorno. La exactitud en altimetría encontrada es aceptable en el mapeo de terreno utilizando vehículos aéreos no tripulados.

Existe un ahorro en tiempo de 52.78\%, es decir, es mucho menos de la mitad de tiempo que la del método convencional, lo cual es consistente respecto a lo encontrado por Vorster y Strecha (2013) en ahorro de tiempo, que aseguran 4 veces menor tiempo de ejecución; pero para un área mayor de trabajo, es decir, que existe una relación directa entre el ahorro de tiempo y el área de trabajo, a mayor área de trabajo mayor ahorro de tiempo, debido a que el dron por su capacidad aérea puede realizar un barrido de terreno en menor tiempo.

Existe un ahorro de costo en $28.57 \%$, lo cual es consistente respecto a lo encontrado por Vorster y Strecha (2013). La mayor incidencia de ahorro se debe en gran medida al menor costo hora/hombre por área de levantamiento en campo; debido al mayor rendimiento por operario (un solo operario realizó la labor de una cuadrilla topográfica tomando datos en terreno) y al menor tiempo de toma de datos en campo para la misma área de estudio. 


\section{CONCLUSIONES}

El mapeo de terreno a través de vehículos aéreos no tripulados brinda resultados fiables y productivos comparados con el método convencional. Para el presente estudio bajo condiciones de terreno con poca vegetación y sin obstrucciones de visibilidad de superficie, resulta el método VANT más seguro, más eficiente y más productivo, con la ventaja de que la información obtenida a través de la fotogrametría es en mayor cantidad y con mayor riqueza de datos comparada con el método convencional.

En cuanto a la exactitud comparada con el método convencional según la ASPRS, el error $\mathrm{RMSE}_{\mathrm{r}}=13.16 \mathrm{~cm}$ en planimetría y $\mathrm{RMSE}_{\mathrm{z}}=13.95 \mathrm{~cm}$ en altimetría detectado se encuentra dentro del rango aplicable para su uso en Mapeo Estándar y Trabajo GIS, para planos con escalas a 1:400.

En cuanto al tiempo comparado con el método convencional, se demuestra un ahorro de $52.78 \%$ en la toma de datos, desde la toma de datos hasta el resultado final.

En cuanto al costo comparado con el método convencional, la utilización de menor personal y menor tiempo en la toma de datos en campo, demuestra un aborro de $28.57 \%$ en el costo total en la toma de datos. Además, el ahorro en tiempo y costo es de mayor importancia a medida que aumenta el área de estudio.

\section{REFERENCIAS BIBLIOGRÁFICAS}

ASPRS. 2014. Positional Accuracy Standards for Digital Geospatial Data. Recuperada de http://www.asprs.org/a/society/committees/standards/Positional_Accuracy_ Standards.pdf

García Julián. 2015. Recuperada del Blog. <http:/ / fotogrametriapractica.blogspot.pe> [Consulta: 21-12-2015]

Siebert, Sebastián y Jochen Teizer. 2013. Mapeo 3D Móvil para levantamiento de movimientos de tierra usando vehículos aéreos no tripulados. School of Civil and Environmental Engineering. Georgia. USA. <http://www.iaarc.org/ publications/fulltext/isarc2013Paper363.pdf $>$ [Consulta: 21.01.2016].

Vorster, Craig y Christoph Strecha. 2013. Exactitud, Aborro de Tiempo y Seguridad UAS para Minería. La Revista Global de Geomática. Edicion UAS. Volumen 27. pp. 22-25. Geomares Publishing. GIM International. <http://pix4d.com/wp-content/uploads/2013/04/GIM-International-Article-UAS-special_small.pdf> [Consulta: 07-02-2016]. 
| Yoshiro Crhistian Zuñiga flores

Fecha de recepción: 29 de agosto del 2016

Fecha de aceptación: 12 de diciembre de 2016

\section{Correspondencia}

Yoshiro Crhistian Zuñiga Flores

najashiro@gmail.com 